\title{
Conditioning Inspired Gases for Tracheostomy
}

Tracheostomy is a frequent procedure in hospitalized patients aimed at improving airway clearance, providing airway protection, and facilitating ventilator liberation. ${ }^{1}$ Under most circumstances, tracheostomy is temporary, with decannulation being a primary goal. ${ }^{2}$ In cases in which tracheostomy is permanent for upper-airway pathology or loss of airway control, maintenance of humidification poses a challenge for the health-care team. Choices for conditioning inspired gases for patients with tracheostomy include continuous aerosol (heated or cool), heat-and-moisture exchanger (HME), or heated humidification. Each has advantages and disadvantages with respect to portability, cost, and maintenance.

Bypassing the upper airway via tracheostomy effectively removes the normal heat and humidification and filtration mechanisms of the respiratory tract. Under normal conditions, gas that reaches the tracheal bifurcation has reached a temperature of $32-34^{\circ} \mathrm{C}$ and $90-$ $100 \%$ relative humidity. ${ }^{3,4}$ This physiologic understanding informs us of the requirements of devices used to replace the heat and moisture functions of the normal upper airway. The requirements for humidification may change with active infection, copious or thickened secretions, alterations in body temperature, and location of care. In the latter, mobile patients are unable to carry heated humidification for obvious logistic reasons.

For many years, the standard of care for providing humidity to patients who had a tracheostomy in the hospital was cool or heated aerosol via a T-piece or tracheostomy mask. In these instances, care and maintenance of the aerosol system (refilling reservoirs and emptying condensate) represented a significant work load for the bedside staff. Condensate also presented a source of potential colonization and infection. ${ }^{5}$ The first HMEs were developed for patients with a tracheostomy to alleviate these problems. ${ }^{6}$ However, HMEs always result in a net moisture loss from the respiratory system

\footnotetext{
Mr Branson has disclosed a relationship with Aerogen, Philips, and Ventec LifeSystems. He is also the Editor-in-Chief of ResPiratory CARE. Ms Gomaa has no conflicts of interest to disclose.
}

Correspondence: Dina Gomaa, Division of Trauma and Critical Care, Department of Surgery, University of Cincinnati Medical Center. E-mail: gomaada@ucmail.uc.edu.

DOI: $10.4187 /$ respcare. 06893 and cannot add moisture in patients with thick secretions where additional moisture might be warranted.

In this issue of the Journal, Nakanishi et $\mathrm{al}^{7}$ describe a comparison of HME and heated humidity in a small

\section{See the Original Study on Page 130}

group of spontaneously breathing subjects with tracheostomy. Specifically, they use the system commonly used for high-flow nasal cannula (HFNC) to provide gas at alveolar conditions to the airway. They demonstrated that active humidity provided an additional $10 \mathrm{mg} \mathrm{H}_{2} \mathrm{O} / \mathrm{L}$ of water vapor compared with the HME. Of note, both devices deliver $100 \%$ relative humidity. The function of an HME allows high relative humidity at a lower temperature, consequently, delivering a lower absolute humidity.

In comparing these 2 systems, it may be tempting to say that a heated humidification system is superior to an HME. With regard to absolute humidity, this would be true. These same authors compared these devices in a bench study and found that the clinical measures were similar to those found in a lung model. ${ }^{8}$ But mobility is impossible for the heated system, and patients with a permanent tracheostomy are often ambulatory. In this regard, the HME is superior or, at a minimum, has advantages. Other important comparisons include cost and infection risk. In our health system, the cost to the hospital for a circuit for HFNC (minus the cannula) is approximately $\$ 42$ compared with $\$ 4$ for a tracheostomy HME. This 10 -fold increase in costs should confer some measurable outcomes.

McNamara et $\mathrm{al}^{9}$ evaluated the use, at home, of heated humidification versus an HME in children with tracheostomy. They reported that, in the short term, heated humidity improved ventilation, likely due to reduced dead space and, in the long term (10 wk), resulted in fewer adverse clinical events. Adverse events include the requirement for tube changes, tube blockage, the need for additional suctioning, and use of saline solution to loosen secretions. In this small study with limited observations, we see the kind of advantages that might justify the cost differences. These authors followed up this study with a study of parents caring for children with tracheostomy and their perceptions of humidification devices. ${ }^{10}$ This interesting study highlights some of these issues. Par- 
ents "balance" the device they choose based on need of the child, secretion removal, comfort, and mobility. ${ }^{10}$ These findings indicate that, for a given patient, the HME is the best choice for mobility versus the heated system for night use of in subjects with limited mobility. ${ }^{10}$ Cost cannot be the only consideration; in many cases, patients may need both.

Stripoli et $\mathrm{al}^{11}$ published a recent study of high-flow oxygen therapy in patients with tracheostomy and at risk for weaning failure. When focusing on work of breathing, they did not find the changes that have been seen with HFNC with regard to breathing frequency or indices of respiratory work. This is easily explained because tracheostomy placement already bypasses the upper-airway dead space, which HFNC washes out. In this trial, the authors did not mention if the tracheostomy tube cuff was inflated or deflated, which might impact findings.

The work by Nakanishi et $\mathrm{al}^{7}$ provided important data and food for thought. The challenge of airway care and conditioning inspired gases in patients with tracheostomy requires an understanding of the physiology and logistics of care. We can find no data that support the use of aerosol delivery to patients with tracheostomy as a method of conditioning inspired gases. In fact, the issues with rainout have negative consequences for infection risk and cost of care. For ambulatory subjects, the HME has clear advantages. The use of heated humidity at night and in patients who are sicker makes physiologic sense and has merit as the method of choice in these subjects. Future studies should evaluate important outcomes to justify the additional cost. In the current health-care climate, cost cannot be the only measure, patient requirements have to play a prominent role.

\section{Dina Gomaa \\ University of Cincinnati College of Medicine Surgery-Trauma Research Cincinnati, Ohio}

\author{
Richard D Branson \\ Editor-in-Chief, ResPiRATORY CARE \\ Irving, Texas \\ University of Cincinnati \\ Cincinnati, Ohio
}

\section{REFERENCES}

1. Cheung NH, Napolitano LM. Tracheostomy: epidemiology, indications, timing, technique, and outcomes. Respir Care 2014;59(6):895915, discussion 916-919.

2. Hess DR, Altobelli NP. Tracheostomy tubes. Respir Care 2014; 59(6):956-971, discussion 971-973.

3. Inglestedt S. Studies of the conditioning of air in the respiratory tract. Acta Otolaryngol Suppl 1956;131:1-81.

4. Walker JE, Wells RE Jr, Merrill EW. Heat and water exchange in the respiratory tract. Am J Med 1961;30:259-267.

5. Thomachot L, Viviand X, Arnaud S, Vialet R, Albanese J, Martin C. Preservation of humidity and heat of respiratory gases in spontaneously breathing, tracheostomized patients. Acta Anaesthesiol Scand 1998;42(7):841-844.

6. Koch $\mathrm{H}$, Allander $\mathrm{C}$, Ingelstedt $\mathrm{S}$, Toremalm NG. A method for humidifying inspired air in posttracheotomy care. Ann Otol Rhinol Laryngol 1958;67(4):991-1004.

7. Nakanishi N, Oto J, Itagaki T, Onodera M, Nishimura M. Humidification performance of passive and active humidification devices within a spontaneously breathing tracheostomized cohort. Respir Care 2019;64(2):130-135.

8. Chikata Y, Oto J, Onodera M, Nishimura M. Humidification performance of humidifying devices for tracheostomized patients with spontaneous breathing: a bench study. Respir Care 2013; 58(9):1442-1448.

9. McNamara DG, Asher MI, Rubin BK, Stewart A, Byrnes CA. Heated humidification improves clinical outcomes, compared to a heat and moisture exchanger in children with tracheostomies. Respir Care 2014;59(1):46-53.

10. McNamara DG, Dickinson AR, Byrnes CA. The perceptions and preferences of parents of children with tracheostomies in a study of humidification therapy. J Child Health Care 2009;13(3):179197.

11. Stripoli T, Spadaro S, Di Mussi R, Volta CA, Trerotoli P, De Carlo F, et al. High-flow oxygen therapy in tracheostomized patients at high risk of weaning failure. Ann Intensive Care 2019;9(1):4. 